
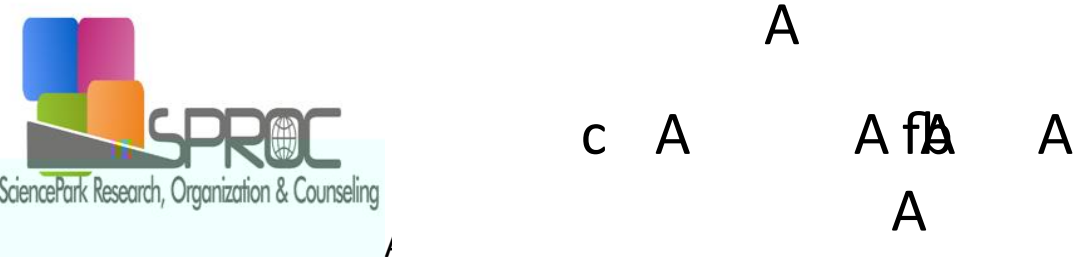

W $\mathrm{n} \quad$ A9;A A;A3129 A3 17A

Ww.gjae.eu

\title{
Impact of acid precipitation on historical monuments and statues
}

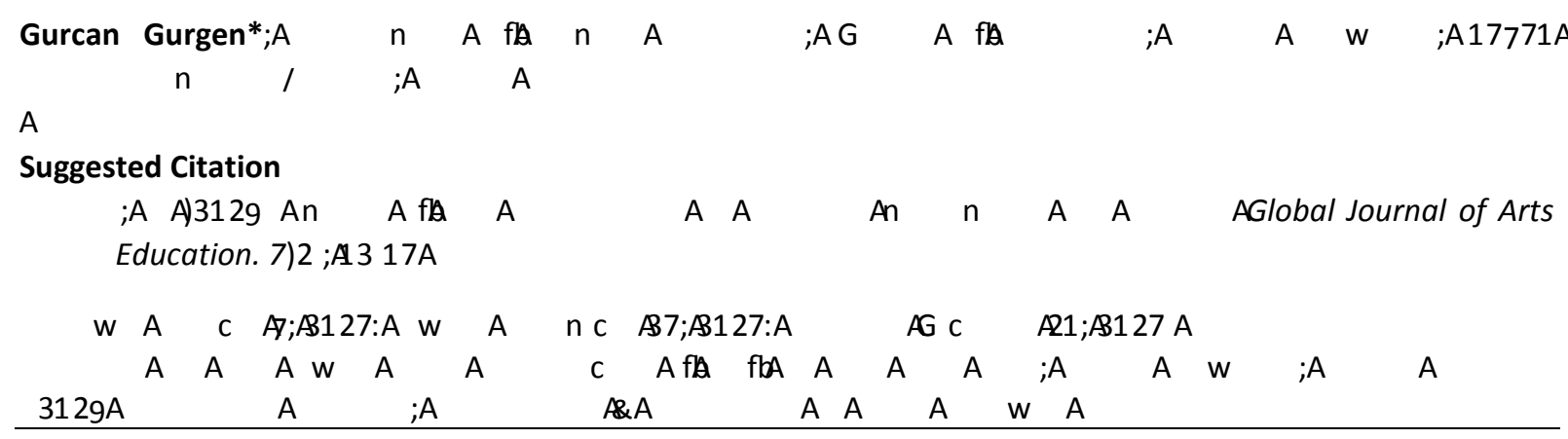

Abstract

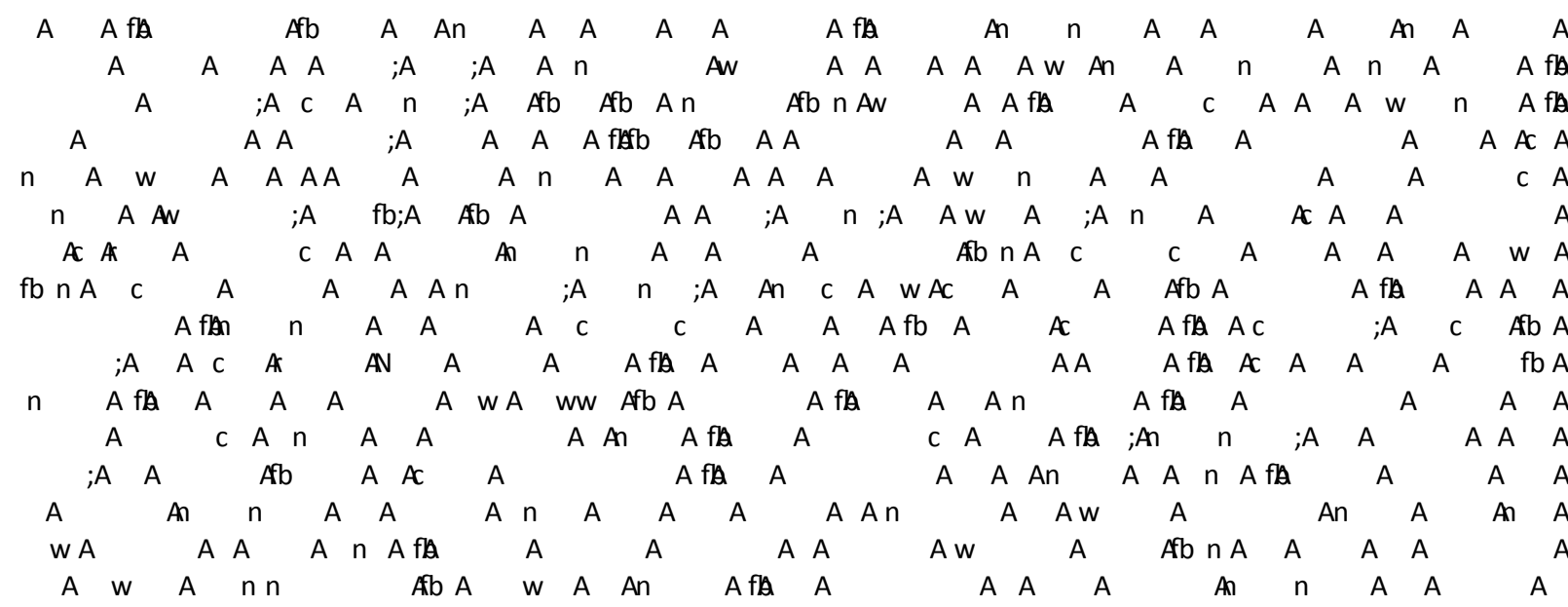

A

A

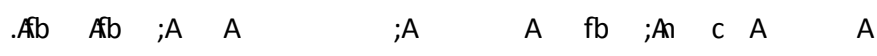

A

A

$A$

A

A

A

A

$A$

A

A

A

AG $A$

w ;A7771;A n
.Gurcan Gurgen;

;A

AE-mail address:A
;AG AfA A 


\section{Introduction}

$\begin{array}{lllllllllll}n & A & A n & n & A & A & A & A & w & y & A f b\end{array} \quad$ A

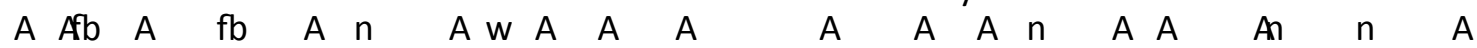

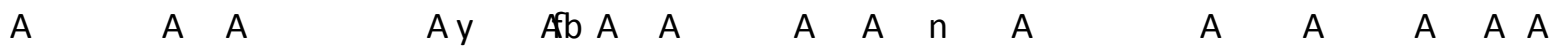

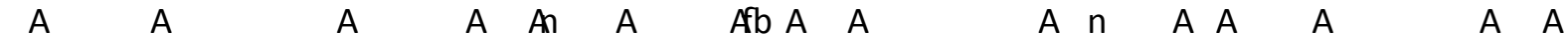

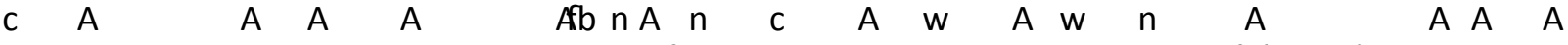

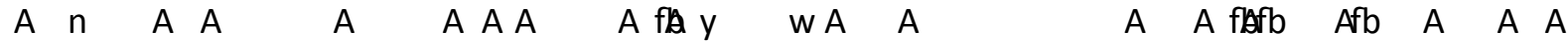
$A C \quad A \quad A \quad A A A$ A $A$ A Ac $n$ A A AC $A$ A $\begin{array}{llllllllllllll}A & A & A & A & A & A & A & A A N & A & A & A & A & A & A\end{array}$ $\begin{array}{llllllllllllll}A & A f t b & A & A & A & w & n & A f t b & A & A & A & A f A & A & \end{array}$

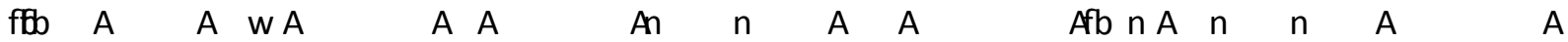
A A A fán A

A

\section{What is acid rain?}

$\begin{array}{llllllllllllll}y & A & A & A & A & A & ; A & A & n & A & A f b & A & A & A \text { faSO }\end{array}$

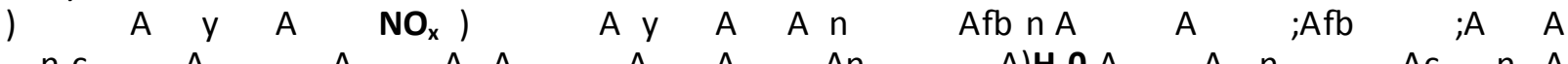

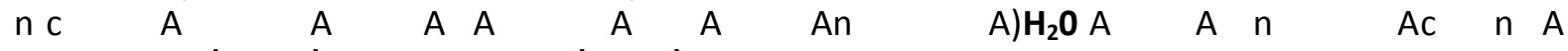
$\begin{array}{lllllllllllll}A & A\left(\mathrm{H}_{2} \mathrm{SO}_{4}\right) & A & A & A\left(\mathrm{HNO}_{3}\right) A & A & A & A & A & A & A & A & A\end{array}$

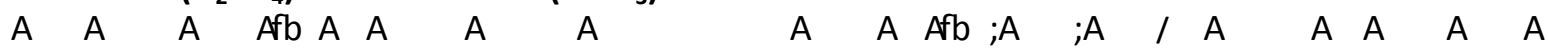

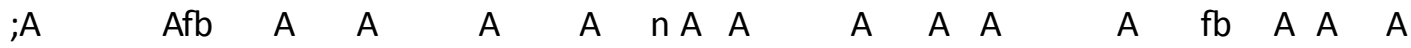
$\begin{array}{llllllllllllll}n & A & C & A \& A & \text { ftb } & ; A 2^{11} 9 & A & A & A & A & n & A & A & A\end{array}$

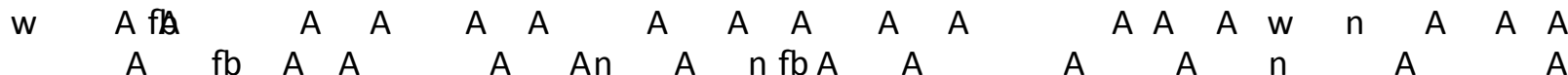
$\begin{array}{llllllllllllllll}n & A A n & A & A & A f t b & A & A & A & A & A & A & A & n A f f & A & A\end{array}$ fb A A A2973AC A $c$ A A A A A c A A A A fla $\quad$ A $A f b$ A A A

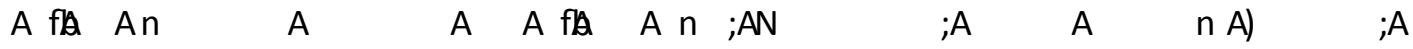
2193 Aw A An A w A A A A $\quad$ A A A A A $A$ A $A$ $\begin{array}{lllllllllllllll}\mathrm{fb} n \mathrm{~A} & \mathrm{~A} & \mathrm{~A} & \mathrm{~A} \text { flafb } & \mathrm{Afb} & \mathrm{A} & \mathrm{A} & \mathrm{A} & \mathrm{Aff} & \mathrm{A} & \mathrm{A} & \mathrm{n} & \mathrm{A} f \mathrm{~A} & \mathrm{~A} & \mathrm{~A}\end{array}$

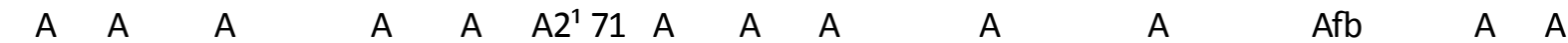

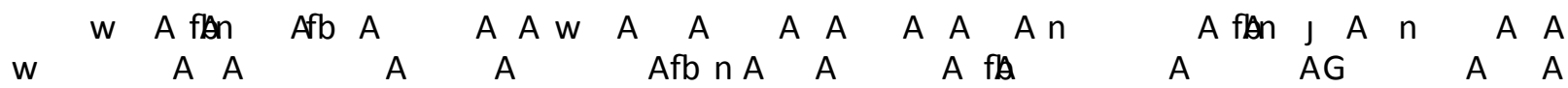

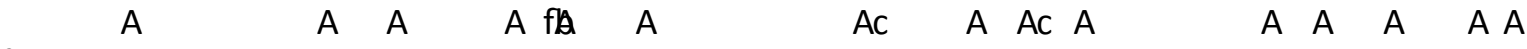
fb $A$ C $\cap A$ AA $\subset$ A $A \cap \cap A$

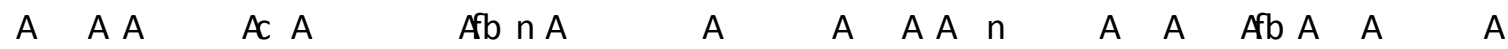
$\begin{array}{lllllllllllllllll} & n & A & A & A & ; A & n & A & A & ; A & A w & A & A & A & A f b & A w & A\end{array}$ A A A A A A A A A A Aw Afla A A $n$ A 7 A A A

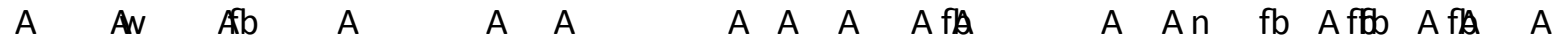

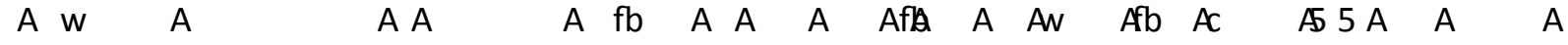

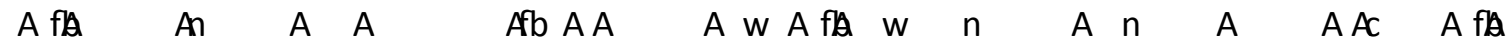
A A A A A $n$ A A A A A A A A A A A A A A $A$

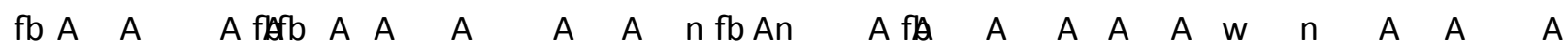

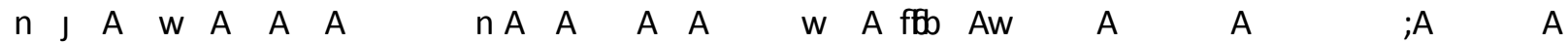

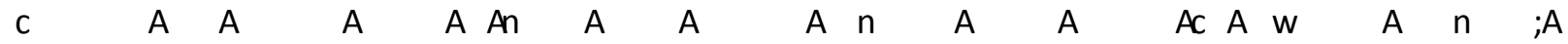
$\begin{array}{llllllllllllllllll}A & A A & A & A & A & A & A & A & A & C & A & y & A & 2 & A & A & A & A\end{array}$

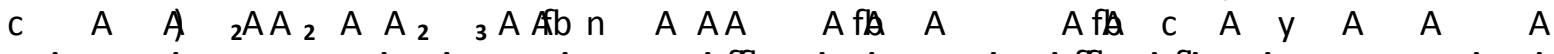

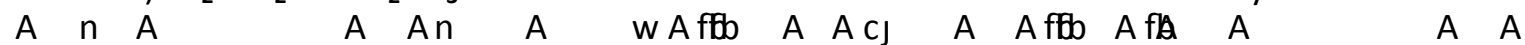
$A n A$ Aftb A

$\begin{array}{lllllllllllllllll}A & A & A & ; A & A & A & A & & A & A & A & & \end{array}$

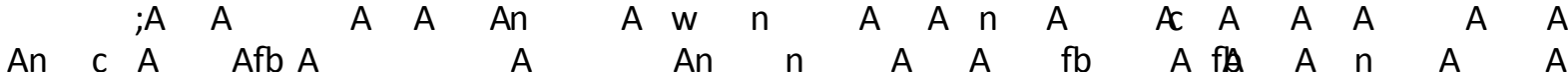

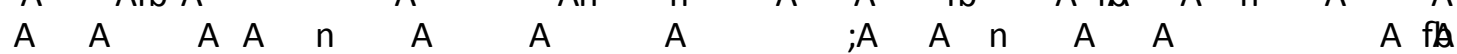

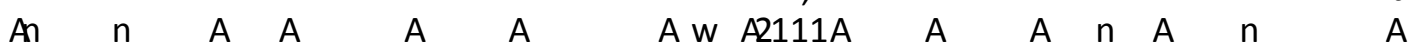

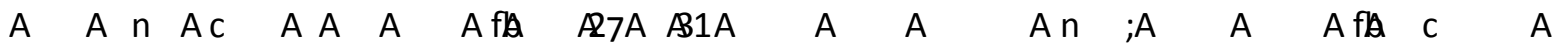




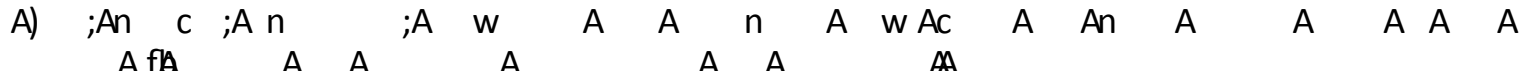
A An $\quad A \quad$ Ab A $\quad A$ C $A$ A $A$ A A

$\begin{array}{llllllll}n & A & \text { An } & \text { An } & & & \\ \text { - } & & \text { Ac } & & A A & A\end{array}$

- A $\quad$ C $A$

- A A

- A A A A A A

- A A A A

- Afto a An Afa A A y A

$\begin{array}{llllllllllllll}A & A & A & ; A & A & A & w & A & A y & w & A & A & A & A\end{array}$ $\begin{array}{lllllllllllllllll}A & A & A & A & A & A & A & A & A & A & A & A & A & A & A & A\end{array}$ $\begin{array}{lllllllllllllllll}A & A & A & A & A & A N & n & ; A & ; A & ; A & A & A & n & A\end{array}$ ) $; A 3127 \quad A N \quad C A \quad A \quad A$ Aw $A \quad$ A An $n \quad A$ w A $n$ A $A$ A n C A fla

A

3. The use of marble

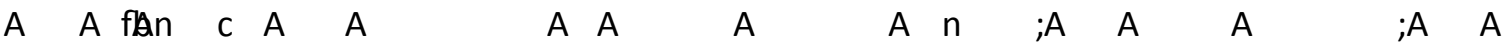

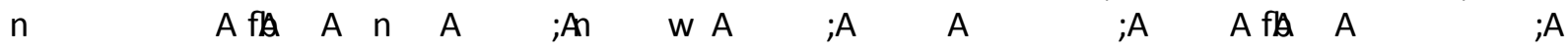
$\begin{array}{llllllllllllllllllllllll}A & A & ; A & A & ; A n & r & ; A & C & A C & A & A f b & A & f A & A & \text { J } & A & A & n & A & A\end{array}$

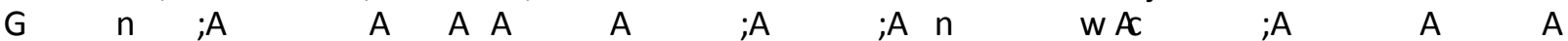

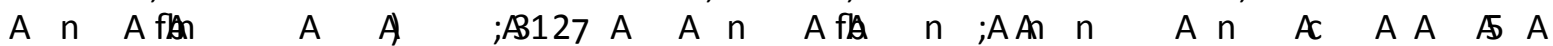
$\begin{array}{llllllllllllllllll}A C & A f b & A & A & A & A & A & A & A & ; A & A & A & A & A & A & A\end{array}$

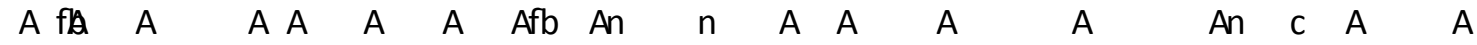

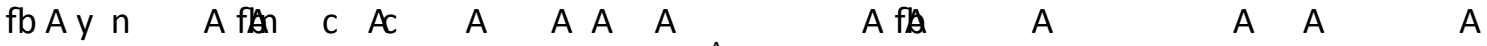

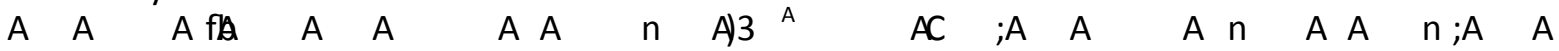

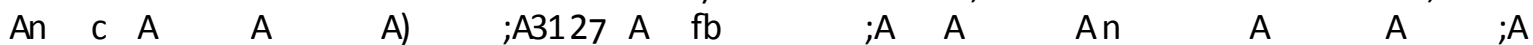

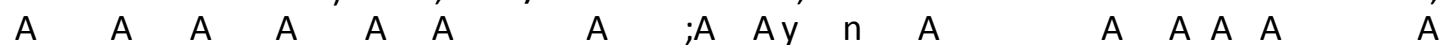

A

\section{How are these works getting destroyed?}

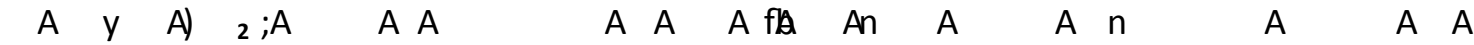
$\begin{array}{lllllllllllllll}A & A & A & A & A & { }_{3} A & A & A & A & A & A & A & A & A\end{array}$

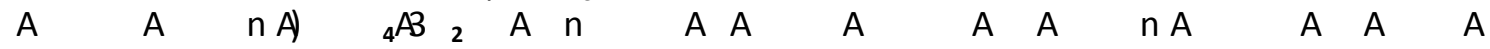
$\begin{array}{lllllllllllllllllllllll} & A & A & ; A & A & A A & ; A & A & A & A & f b & A & n & A & A & A n & c & A & f b & A\end{array}$

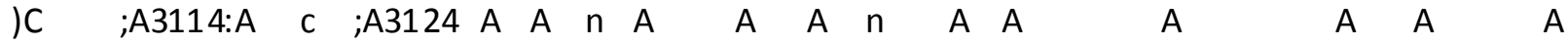
Afb $n$ A C A Aff A A A A A A $n$ Afb A A $A$ $\begin{array}{lllllllllllll}A & A & A & A & n & A & A & A & A & A & A\end{array}$

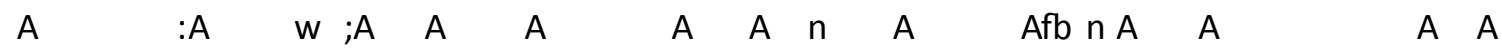

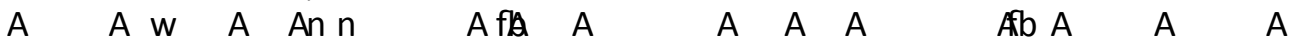

\section{What can be done?}

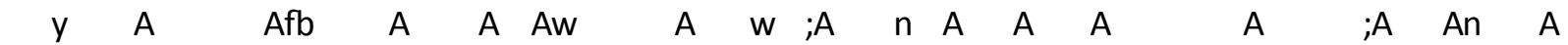

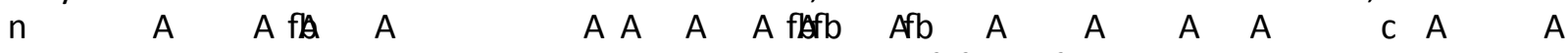

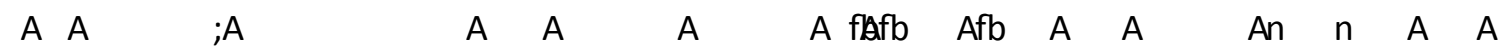

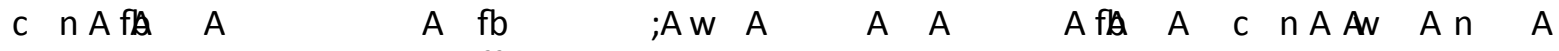
An $n \quad A \quad A$ Av A ftb Aw A $A$ A A $A$ A $\quad$ A A A $A$ A An $n$;A An A A A A A $n$ A A $\begin{array}{lllllllllll}A & A & A & A & ; A & A & A & A & A & A & A\end{array}$ $\begin{array}{llllllllllll}A & A f f a & A & A f b & A & A & A c & A & A & A & A & A f b n A\end{array}$

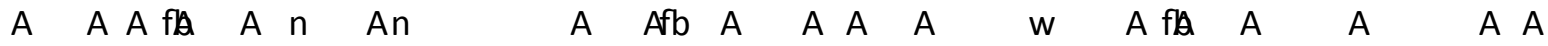


ftb $A \quad A \quad A \quad A \quad A A \quad A A \quad A \quad A$ A f $A$ A A A $A$ A A A $\quad$ A

A A W A A

$$
\text { A } A \quad \text { A } A \text { Ab } \quad A \quad A \quad A C A
$$

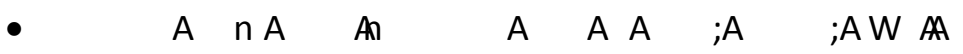

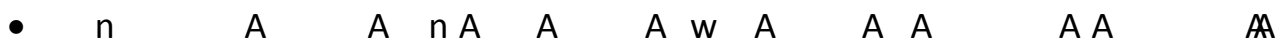

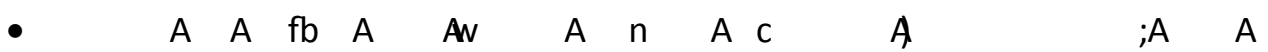

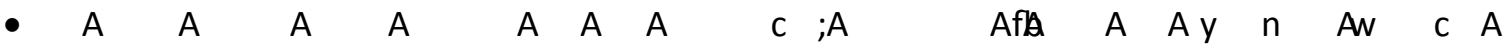
$\mathrm{fb} ; \mathrm{A}$ A $A$ A $A$ A

A

$\begin{array}{cccccccccccccccccccccccccc}A & & & A & A & A & & A & A & & A & A & A & A & & A & n & & A & & A \\ & n & A & A & A & w & A & A & A & w & A b & A & & A & A & A & f b & A & A & A b & A\end{array}$ $A$ A $\quad A$ A $A \cap A b$ A

A

$\begin{array}{lllllllllllllllll}G & A & A & ; A & \mathrm{~A} & A & A & A & A & \text { fb } & n & A & A & A & \text { A }\end{array}$

- A

- A A

- $n$ A A

- A A

A

\subsection{Consolidation}

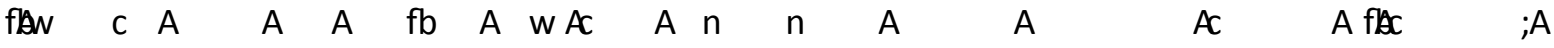

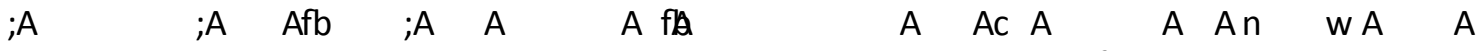
$\begin{array}{lllllllllllllllll}C & ; A & ; A & A W & A & A N & A & A & A & A & A & A & A b A & A & A & A & A\end{array}$

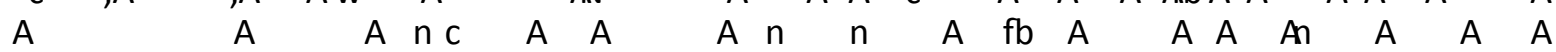
A A A

\subsection{Plastic repair}
A $A A$
A $A$ A
A $A$ AfB An

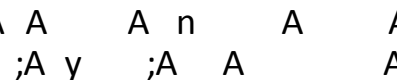
$\mathrm{Afb} \quad \mathrm{A}$

\subsection{Cosmetic repair}

$A \quad f b \quad A \quad A \quad A$ ;A $\quad n \quad A \quad A$ A Afb $r \quad A \quad A$ A fb $\mathrm{A} \quad \mathrm{A}$

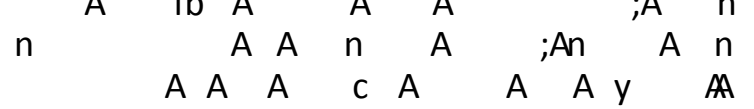

A

\subsection{Application of the laser beam}

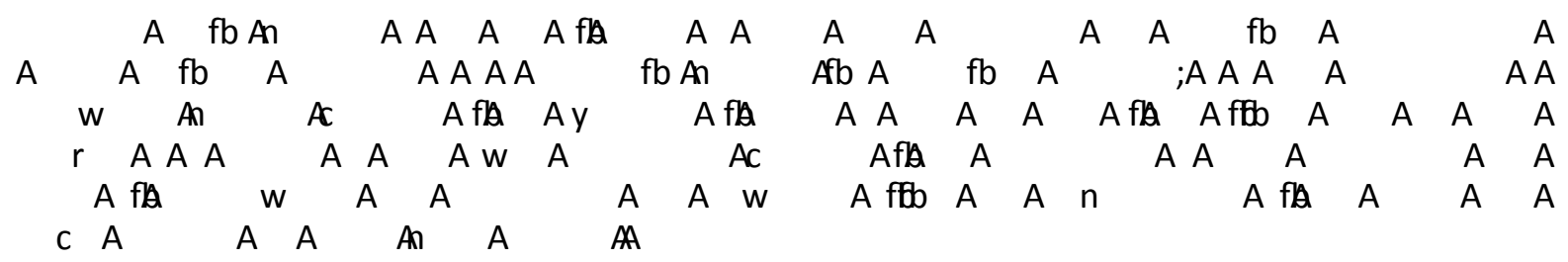

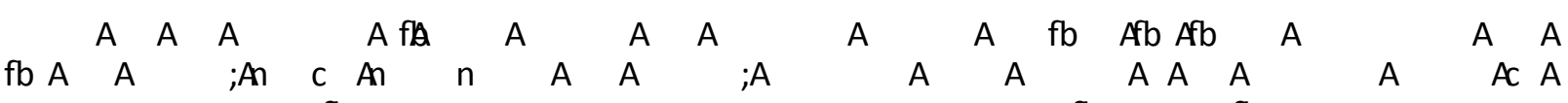

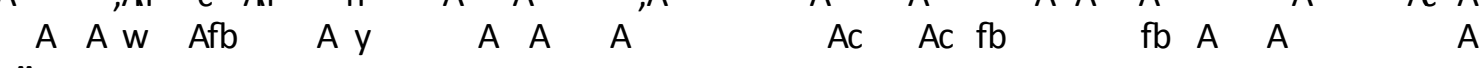



;A A3129 An AfA A
A A
An
A A
A C A
A f A A
(9) 2 ;A3 $17 \mathrm{~A}$

A

\section{References}

C ;A A A ;A\&AC ;AN A A)3114 AN
$A$ fb $A$ y $A$
A A
n A c
AAmerican Chemical Society, 42;A21392145 AA ;A A2 93 A A

A A

A

w AEnvironmental Science and Technology, 16 A

C ;A A3124 A A An J A A flA of Scientific Engineering and Technology, 2)9; $993997 \mathrm{~A}$

c ;A ; $; \& A$ ftb $\quad ; A \quad A 2^{119}$ AExperimental reversal of acid rain effects $A \quad A \quad$.A $\quad A \quad A \& A$ ;A A A 13127 A Mermer yapılarda plastik ve kozmetik onarımlar A w A fb $n$ A

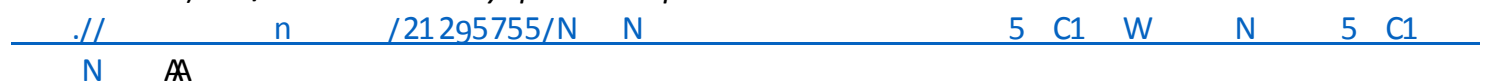

A

A 